\title{
The genus Capparis L. in India
}

\author{
Maurya S., Datar M.N. \& R.K. Choudhary
}

Published by MACS-Agharkar Research Institute, Pune, India

ISBN: 978-81-946051-0-2. Pages 91, Open access: www.aripune.org

The genus Capparis L. in India is a superb production from Agharkar Research Institute in Pune, India. In this work the members of the genus Capparis distributed in four sections are treated: Capparis, Sodada, Busbeckea and Monostichocalyx. This work is a fine and detailed monograph that includes 34 species and a subspecies of this genus present on the Indian subcontinent. The species are represented by beautiful photographs that allow us to appreciate their particular morphological structures and recognize them in detail. It presents an exhaustive review of the published literature of this genus in relation to the taxa present in India, analyzing the sections from the time of De Candolle (1824) and the first study on Capparis in India by Joseph Dalton Hooker (1872), up to the present day. It offers a biogeographic perspective of this genus at a global and subcontinental level, contrasting the last nomenclatural changes made in the New World in recent years and updating the present circumscription of the present Capparis distribution pattern restricted to the Old World.

At the level of subcontinental India and India sensu stricto, the work presents maps in which the distribution patterns of the species and main populations of Capparis are illustrated. The maps are based on georeferenced collections of 14 national and international herbaria, literature reviews, and recent surveys by the authors. This is a useful tool and a significant contribution to understanding biogeographic patterns with positive implications in evolutionary studies and with practical applications in conservation and management. Sixteen of the 34 recorded species are endemic to India, including $C$. cinerea M.Jacobs endemic to Northeast India that is considered to be presumably extinct. In addition, a table is presented that documents the multiple nutritional and medicinal uses of 23 of the 35 taxa of Capparis treated. Among the medicinal uses, its properties are documented to alleviate disorders in the liver, kidneys, skin conditions, antitumor, as an antidote against malaria, diabetes, various inflammations, tuberculosis, heart and neurological problems, ulcers, asthma and cough.

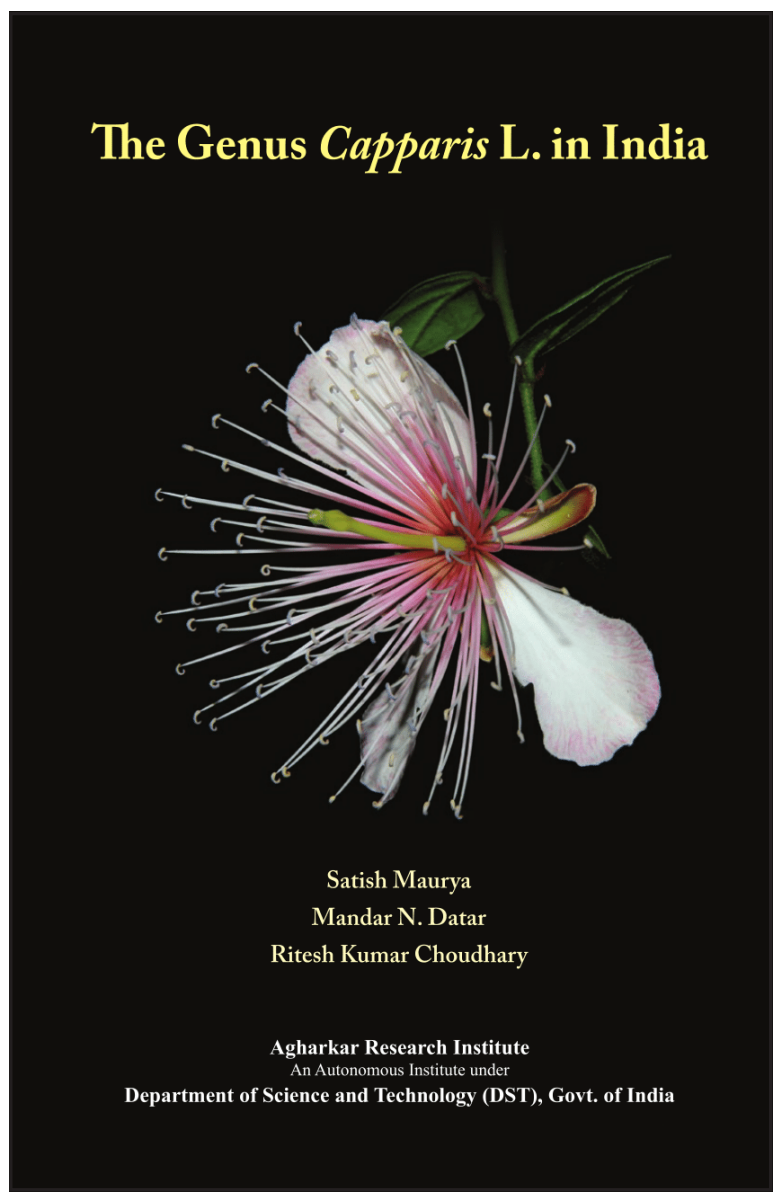


The nomenclature of the taxa follows the most recent treatments (Govaerts 2003, Zhang \& Tucker 2008, Fici 2015, IPNI 2020, Tropicos 2020, and POWO 2020). A taxonomic key is provided for the identification of sections and species based on morphological characters. For each species descriptions, relevant notes, comments of the types, etymology of the scientific names, common names in English, Hindi and Tamil languages, phenology data, distribution map, citation of studied specimens and uses are presented. The illustrations include photos of the habit, details of the branches, closeups of the flowers and of the floral parts in detail, making the book superbly illustrated.

Xavier Cornejo, University of Guayaquil, Ecuador 